\title{
Adaptive Edge Enhancing Technique of Impulsive Noise Removal in Color Digital Images
}

\author{
Bogdan Smolka \\ Silesian University of Technology, Department of Automatic Control, \\ Akademicka 16, 44-100 Gliwice, Poland \\ smolka@ieee.org
}

\begin{abstract}
In this paper a novel class of noise attenuating and edge enhancing filters for color image processing is introduced and analyzed. The proposed adaptive filter design is minimizing the cumulative dissimilarity measure of a cluster of pixels belonging to a sliding filtering window and outputs the most centrally located pixel. The proposed filter is computationally efficient, easy to implement and very effective in suppressing impulsive noise, while preserving image details and enhancing its edges. Therefore it can be used in any application in which simultaneous denoising and edge enhancement is a prerequisite for further steps of the color image processing pipeline.
\end{abstract}

Keywords: color image enhancement, impulsive noise reduction.

\section{Introduction}

During image formation, acquisition, storage and transmission many types of distortions limit the quality of digital images [1, 2, 3]. Quite often, images are corrupted by the impulsive noise caused mainly either by faulty image sensors or due to transmission errors. Common sources of impulse noise include also lightenings, industrial machines, car starters, faulty or dusty insulation of high voltage powerlines and various unprotected electric switches. These noise sources generate short time duration, high energy pulses which block the regular signal, resulting for example in bothering spots on the TV screen and sharp click sounds in the audio [4].

The Vector Median Filter (VMF) is the most popular filter intended for smoothing out spikes injected into the color image by the impulse noise process, [5, 6, 7]. This filter is very efficient at reducing the impulses, preserves sharp edges and linear trends, however it does not retain fine image structures, which are treated as noise and therefore generally the VMF tends to produce blurry images. This unwanted feature of the VMF is quite important as much of the image information is contained in its edges and sharp edges are pleasing to humans and are desirable for machine processing [8,9, 10]. As a result much research has been devoted to the construction of filters which can cope with image noise while simultaneously preserving image details and enhancing image edges. 
In this paper a solution to the problem of image noise filtering with edge enhancing abilities is presented. Extending the VMF concept using the peer groups introduced in 11, 12, it is possible to efficiently remove impulse noise while sharpening the color image edges. The proposed filtering design excels over the VMF, preserves much better image details and produces images with sharp object boundaries. Additionally, the proposed design ia able to adapt to the noise intensity level so that no tuning parameters are required.

\section{Vector Median Filter}

In this paper the color image is defined as a two-dimensional matrix of size $N_{1} \times N_{2}$ consisting of pixels $\boldsymbol{x}_{i}=\left(x_{i 1}, x_{i 2}, x_{i 3}\right)$, indexed by $i$, which gives the pixel position on the image domain. Components $x_{i k}$, for $i=1,2, \ldots, N, N=N_{1} \cdot N_{2}$ and $k=1,2,3$ represent the color channel values quantified into the integer domain.

As color images are highly non-stationary, the filtering operators work on the assumption that the local image features can be extracted from a small image region centered at pixel $\boldsymbol{x}_{i}$ called a sliding filtering window, $W$. Thus, the output of the filtering operation will depend only on the samples from the filtering window. Of course, the size and shape of the window influence the properties and efficiency of the image processing operations and are therefore application dependent. However, in order to preserve image details, mostly a $3 \times 3$ window is used to process the central pixel surrounded by its neighbors.

To remove the impulse noise various filtering approaches based on the order statistics theory have been devised. The most popular filtering class operating on a sliding window is based on the reduced or aggregated ordering which assigns an aggregated dissimilarity measure to each color pixel from the filtering window [1,13. The aggregated dissimilarity measure assigned to pixel $\boldsymbol{x}_{i}$ is defined as

$$
R_{i}=\sum_{j=1}^{n} \rho\left(\boldsymbol{x}_{i}, \boldsymbol{x}_{j}\right), \quad \boldsymbol{x}_{i}, \boldsymbol{x}_{j} \in W
$$

where $\rho(\cdot)$ is the chosen dissimilarity measure. The scalar accumulated dissimilarity measures are then sorted and the vectors are correspondingly ordered

$$
R_{(1)} \leq \ldots \leq R_{(n)} \Rightarrow \boldsymbol{x}_{(1)} \leq \ldots \leq \boldsymbol{x}_{(n)} .
$$

The dissimilarity measure depends on the kind of relationship between the sample vectors used to measure their difference. Usually the distance between vectors and the angle between them is utilized. The combination of the magnitude and directional processing can also be used, [14, 15.

In this paper we will focus on the vector median filter defined using the accumulated sum of distances between vectors, which serves as a dissimilarity measure. The vector median of a set of vectors belonging to a filtering mask $W$ is defined as the vector $\boldsymbol{x}_{(1)}$ for which the sum of distances to all other vectors from $W$ is minimized, [5, 16, 17, 18

$$
\boldsymbol{x}_{(1)}=\underset{\boldsymbol{x} \in W}{\arg \min } \sum_{j=1}^{n}\left\|\boldsymbol{x}-\boldsymbol{x}_{j}\right\|,
$$




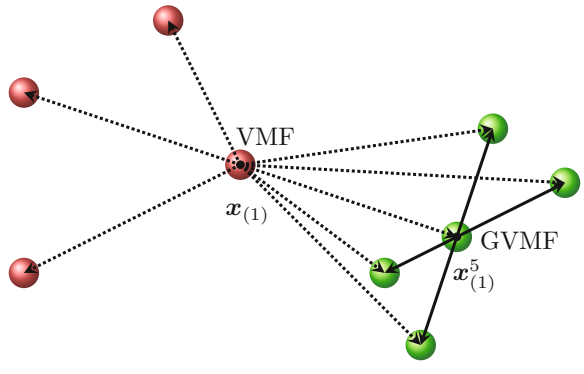

(a)

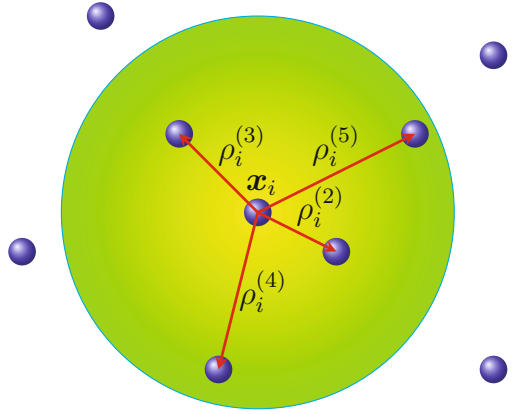

(b)

Fig. 1. Illustration of the peer group concept: (a) output of the VMF, $(n=9)$ and GVMF for $\alpha=5$ and (b) the peer group associated with $\boldsymbol{x}_{i}, \alpha=5$

where $\|\cdot\|$ denotes the Euclidean distance. The construction of the VMF is illustrated in Fig. 1a, where the Euclidean distance is used. As can be seen, the vector median of the set of pixels is centrally located within the samples from the filtering window, meaning that the sum of distances to all other samples from $W$ is minimized.

\section{Generalized Vector Median Filter}

As in the definition of the VMF the sum of distances is used (Eqs. 11 3), we can say that the vector median is the vector $\boldsymbol{x}_{(1)}$ whose average distance to the $n$ vectors from $W$ is minimized. So we see that the vector median and also the scalar median is utilizing the concept of averaging, which is a little bit surprising, taking into account its properties, which are clearly opposed to the averaging based filters.

In this paper we propose to generalize the definition of the vector median. In the new approach the vector median will be the vector $\boldsymbol{x}_{(1)}^{\alpha}$ for which the sum of $\alpha$ smallest distances to other vectors from $W$ is minimized. For $\alpha=n$ the output of the Generalized VMF (GVMF) is identical with the standard VMF and for $\alpha=1$ the identity filter is obtained, as the smallest distance is always zero, because it is the distance of the reference pixel to itself.

If the distance between the vector $\boldsymbol{x}_{i}$ and $\boldsymbol{x}_{j}$ is denoted as $\rho_{i, j}$, then we can order the set of distances $\rho_{i, j}$, for $j=1, \ldots, n$ and obtain the following sequence: $\rho_{i}^{(1)} \leq \ldots \leq \rho_{i}^{(\alpha)} \leq \ldots \leq \rho_{i}^{(n)}$, where $\rho_{i}^{(k)}$ is the $k$-th smallest distance from $\boldsymbol{x}_{i}$ and $\rho_{i}^{(1)}=\left\|\boldsymbol{x}_{i}-\boldsymbol{x}_{i}\right\|=0$. For each pixel in the filtering window the cumulated sum $R_{i}^{\alpha}$ is calculated

$$
R_{i}^{\alpha}=\sum_{k=1}^{\alpha} \rho_{i}^{(k)},
$$

and the output of the generalized VMF is the pixel for which the trimmed sum of distances $R^{\alpha}$ is minimized. 


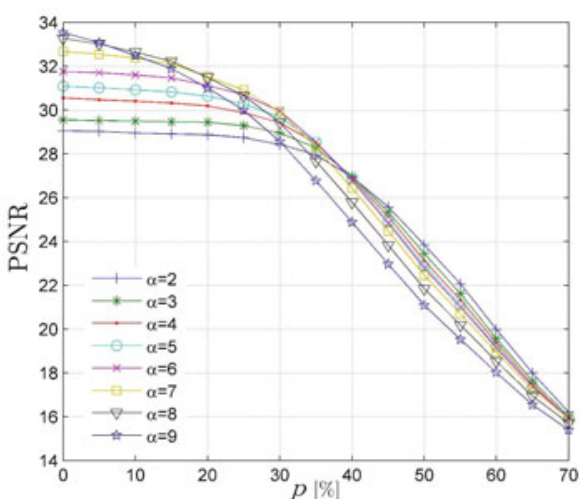

(a) PSNR, noise model U

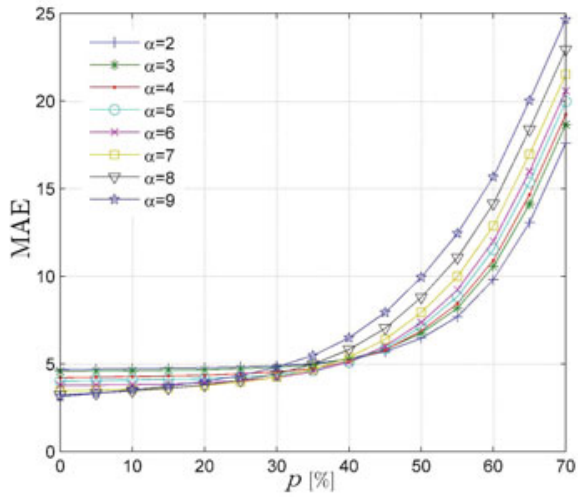

(c) MAE, noise model U

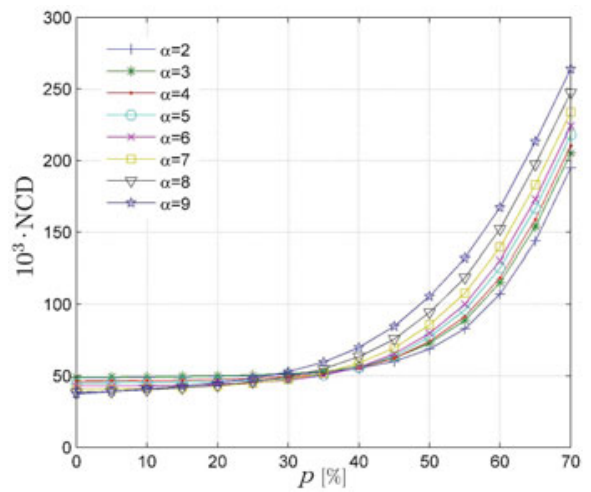

(e) NCD, noise model U

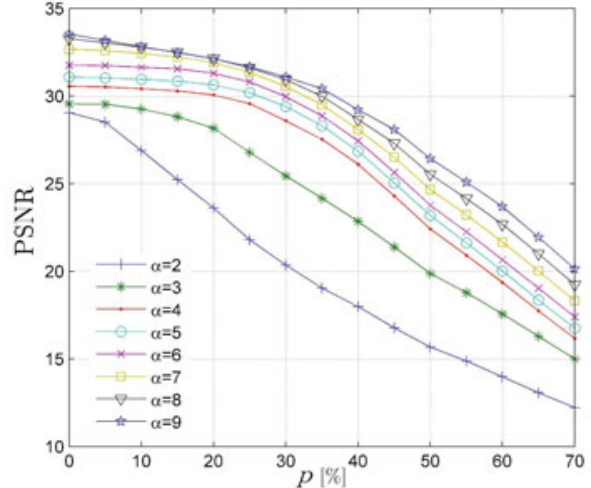

(b) PSNR, noise model I

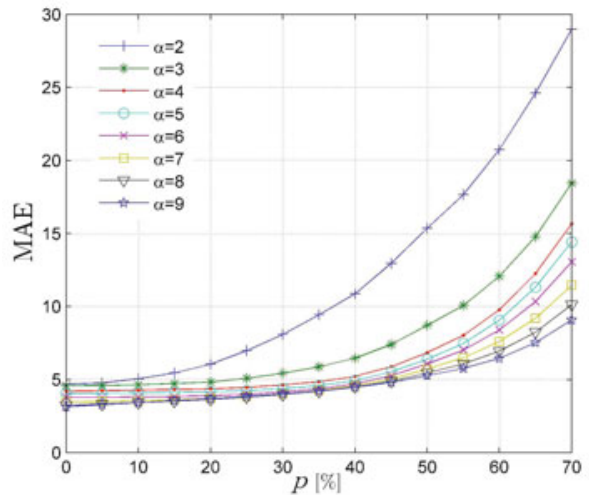

(d) MAE, noise model I

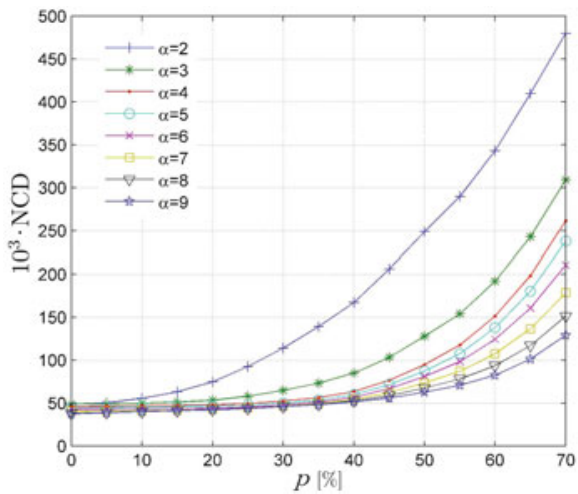

(f) NCD, noise model I

Fig. 2. Dependence of the PSNR (a, b), MAE (c, d) and NCD (e, f) quality measures on the contamination intensities for the used noise models $\mathrm{U}$ and I applied to the LENA test image filtered with the GVMF for various values of the $\alpha$ parameter 
In [11] a concept of the so called peer group filtering was introduced. This concept can be used to describe the construction of the proposed filtering approach. The peer group $\mathcal{P}(\boldsymbol{x}, \alpha)$, denotes the set of $\alpha$ pixels consisting of $\boldsymbol{x}$ and $(\alpha-1)$ nearest pixels belonging to $W$. Using the peer group concept we can define the generalized vector median filter output as the sample $\boldsymbol{x}$ whose peer group of size $\alpha$ has the smallest accumulated sum of distances $R^{\alpha}$, (see Fig. 1 b). In other words the output of the GVMF is the pixel centrally located within a peer group of pixels with minimal dispersion, expressed as the sum of distances.

It is worth noticing the similarity of the new filter design with the $\alpha$-trimmed vector median filter. The trimming operation in the $\alpha$-trimmed filter is however being performed on the ordered set of vectors, whereas in the construction of the new filter, the trimming is applied to the ordered set of distances associated with a pixel from the filtering window.

So, the new filtering design is utilizing the concept of a peer group which has been already successfully utilized for impulse noise removal [19,20, 21] and can be regarded as a generalization of the vector median filter, which is obtained as a special case of the new filtering technique.

\section{Adaptive Design}

As can be observed in Fig. 2, the quality measures, which will be defined later together with the used noise models, depend significantly on the $\alpha$ parameter of the GVMF, which evokes the need for an adaptive scheme of adjusting this parameter to the local image structures.

In 22 the following criterion for choosing the $\alpha$ parameter was proposed

$$
\alpha=\max \alpha^{\prime} \quad \text { subject to }\left(\sum_{l=1}^{\alpha^{\prime}} \rho_{j}^{(l)}\right) \leq \rho_{j}^{(n)}, j=1, \ldots, n,
$$

where $\rho_{j}^{(n)}$ is the largest distance between the central pixel $\boldsymbol{x}_{j}$ and its neighbors from $W$. This rule for the setting of the $\alpha$ values works well for pixels corrupted by impulsive noise, however for uncorrupted pixels in homogeneous image areas, usually the number of the pixels $\alpha$ in the peer-group is very small, as the distances between pixels are comparable. Therefore a new scheme for the adaptive determination of the choosing the $\alpha$ value has been elaborated.

The adaptive algorithm which will be denoted as Adaptive Generalized VMF (AGVMF) requires to calculate for each pixel $\boldsymbol{x}_{k}$, the distances $\rho_{k, l}, l=1, \ldots, n$, $l \neq k$ to other pixels belonging to the filtering window. The largest distance $\rho_{k}^{(n)}$ is used for building the peer-groups $\mathcal{P}\left(\boldsymbol{x}_{\boldsymbol{k}}, \alpha_{k}\right)$ which consist of $\alpha_{k}$ pixels contained in a sphere centered at $\boldsymbol{x}_{k}$ with diameter $\rho_{k}^{(n)}$. In this way the peer group consists of $\alpha_{k}$ pixels $\boldsymbol{x}_{l}$ satisfying: $\rho_{k, l} \leq \rho_{k}^{(n)} / 2$. The highest value of the $\alpha_{k}$ for $k=1, \ldots, n$, denoted as $\alpha$ serves as an self-adaptive parameter of the proposed filter. Thus, the filter output is the pixel $\boldsymbol{x}_{k}$ for which the aggregated, trimmed distance $R_{k}^{\alpha}$ defined in (4) is minimized

$$
k=\underset{j}{\arg \min } \sum_{l=1}^{\alpha} \rho_{j}^{(l)}, \quad j=1, \ldots, n, \quad \text { where }
$$




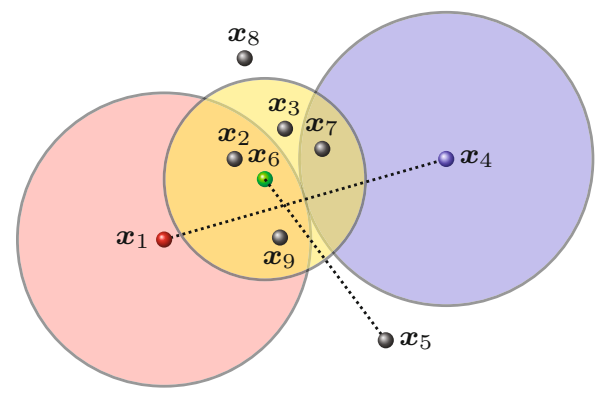

(a)

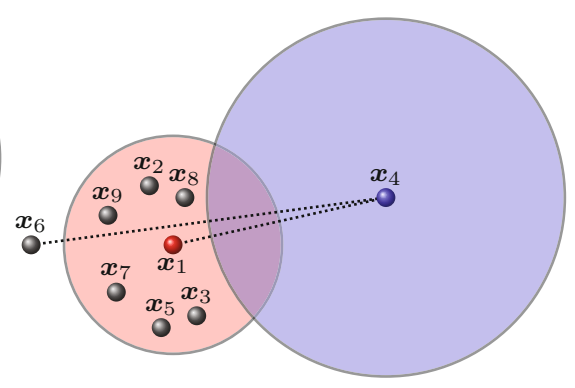

(b)

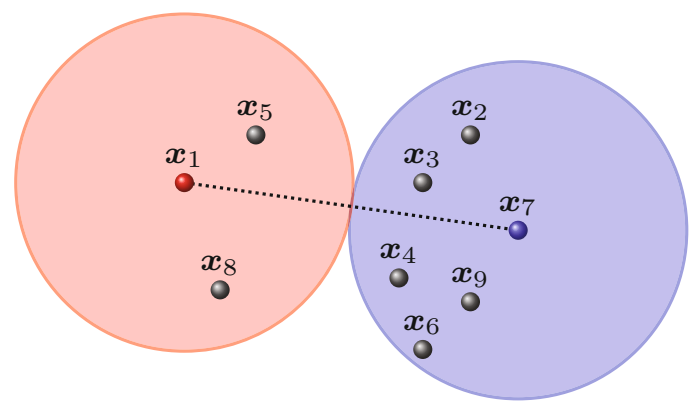

(c)

Fig. 3. Illustration of the adaptive determination of the maximum size cluster

$$
\alpha=\underset{l}{\arg \max } \rho_{j}^{(l)} \leq \frac{1}{2} \rho_{j}^{(n)}, \quad j=1, \ldots, n .
$$

Figure 3 shows an exemplary configuration of pixels. For the pixel $\boldsymbol{x}_{1}$ the most distant neighbor is the outlying pixel $\boldsymbol{x}_{4}$ and in the sphere centered at $\boldsymbol{x}_{1}$ with the diameter equal to the distance $\rho_{1,4}$ four pixels are contained. The peer group of pixel $\boldsymbol{x}_{4}$, whose most distant neighbor is $\boldsymbol{x}_{1}$ contains 2 pixels and the largest peer group consisting of 5 pixels is assigned to pixel $\boldsymbol{x}_{6}$ whose most distant pixel is $\boldsymbol{x}_{5}$. In this way the proposed filter is searching for a cluster of $\alpha=5$ pixels with the lowest trimmed sum of distances.

As can be observed in Fig. 3 $\mathrm{p}$ the proposed design is able to cope with the outliers introduced by the noise process as their peer groups do not contain any other pixels or like in a situation depicted in Fig. 33 the peer group size is quite low.

As often a few clusters with the same maximum number of pixels is found, then the pixel centrally located in the most compact cluster is chosen as the filter output. In other words, the output is the center of the peer-group whose aggregated distances to its members attains a minimum value.

Additionally, in order to preserve image details a switching mechanism has been incorporated into the adaptive filter design. As can be observed, the 
adaptive filter removes efficiently the impulses and enhances the image edges. The changes introduced into the image are significant at pixels corrupted by impulse noise and at edges. If the differences between the channel components of the original and filtered image pixels are small, then most probably these pixels are not corrupted and do not belong to an edge.

If $\boldsymbol{x}_{i}=\left\{x_{i 1}, x_{i 2}, x_{i 3}\right\}$ denotes a pixel at a position indexed by $i$ and $\boldsymbol{y}_{i}=$ $\left\{y_{i 1}, y_{i 2}, y_{i 3}\right\}$ is the output of the Adaptive Generalized VMF (AGVMF), then the final output will be $\boldsymbol{x}_{i}$ if $\left|x_{i k}-y_{i k}\right|<\delta$ for $k=1,2,3$ and $\boldsymbol{y}_{i}$ otherwise. In other words, if the changes in the RGB components are less than a threshold $\delta$ then the color image pixels are not changed, otherwise they are replaced by the AGVMF. Extensive experiments indicate that the value of $\delta$ equal to 30 provides a good compromise between detail preservation and noise reduction.

\section{Experiments}

For the evaluation of the efficiency of the proposed adaptive denoising design a set of standard color images has been contaminated with two noise types. In the first model, which will be called impulsive salt and pepper noise model denoted as I, the noisy signal is modeled as $\boldsymbol{x}_{i}=\left\{x_{i 1}, x_{i 2}, x_{i 3}\right\}$, where

$$
x_{i k}=\left\{\begin{array}{l}
\rho_{i k} \quad \text { with probability } \pi, \\
o_{i k} \quad \text { with probability } 1-\pi,
\end{array}\right.
$$

where $\boldsymbol{o}_{i}$ is the original, uncorrupted image pixel and the contamination component $\rho_{i k}$ is a random variable, which takes the value 0 or 255 with the same probability. In this noise model the contamination of the color image components is uncorrelated and the overall contamination rate is $p=1-(1-\pi)^{3}$.

The second type of noise, called random-valued or uniform noise denoted as $\mathrm{U}$ is modeled as

$$
\boldsymbol{x}_{i}=\left\{\begin{array}{l}
\boldsymbol{\rho}_{i} \quad \text { with probability } p, \\
\boldsymbol{o}_{i} \quad \text { with probability } 1-p,
\end{array}\right.
$$

where $\boldsymbol{\rho}_{i}$ is a noisy pixel with all channels corrupted by noise of uniform distribution in the range $[0,255]$. In the first model the noise can corrupt one, two or all three channels. In the second all channels are contaminated by random values within the range $[0,255]$.

For the measurement of the restoration quality the commonly used Root Mean Squared Error (RMSE) expressed through the Peak Signal to Noise Ratio (PSNR) was used as the RMSE is a good measure of the efficiency of impulsive noise suppression. For the evaluation of the detail preservation capabilities of the proposed filtering design the Mean Absolute Error (MAE) has been used.

The PSNR, which measures the impulsive noise removal efficiency of a filter, is defined as

$$
\operatorname{PSNR}=20 \log _{10}\left(\frac{255}{\sqrt{M S E}}\right), \quad \operatorname{MSE}=\frac{1}{N} \sum_{i=1}^{N}\left\|\boldsymbol{x}_{i}-\boldsymbol{o}_{i}\right\|_{2}^{2},
$$


where $N$ is the total number of image pixels, and $x_{i k}, o_{i k}$ denote the $k$-th component of the noisy image pixel channel and its original, undisturbed value at a pixel position $i$, respectively. The MAE defined as

$$
\mathrm{MAE}=\frac{1}{N} \sum_{i=1}^{N}\left\|\boldsymbol{x}_{i}-\boldsymbol{o}_{i}\right\|_{1}
$$

where $\|\cdot\|_{\gamma}$ denotes the $L_{\gamma}$ Minkowski norm, is a good measure of detail preservation.

Since RGB is not a perceptually uniform space, in the sense that differences between colors in this color space do not correspond to color differences perceived by humans, the restoration errors were also analyzed using the Normalized Color Difference (NCD) based on the CIE Lab color space, [1].

$$
\begin{gathered}
\Delta E=\frac{1}{N} \sum_{i=1}^{N} \sqrt{\left(L_{\mathbf{o}_{i}}^{*}-L_{\mathbf{x}_{i}}^{*}\right)^{2}+\left(a_{\mathbf{o}_{i}}^{*}-a_{\mathbf{x}_{i}}^{*}\right)^{2}+\left(b_{\mathbf{o}_{i}}^{*}-b_{\mathbf{x}_{i}}^{*}\right)^{2}}, \\
N C D=\frac{N \Delta E_{1}}{\sum_{i=1}^{N} \sqrt{\left(L_{\mathbf{o}_{i}}^{*}\right)^{2}+\left(a_{\mathbf{o}_{i}}^{*}\right)^{2}+\left(b_{\mathbf{o}_{i}}^{*}\right)^{2}}},
\end{gathered}
$$

where $L^{*}$ represents lightness values and $\left(a^{*}, b^{*}\right)$ chrominance values corresponding to original $\mathbf{o}_{i}$ and noisy (filtered) $\mathbf{x}_{i}$ samples expressed in CIE LAB color space.

In order to evaluate the edge enhancing and noise canceling properties of the proposed filter a synthetic color test image has been prepared, (Fig. 4a). This image has been blurred by rotating it clockwise about 3 degrees, applying moving average smoothing and rotating back about 3 degrees. The rotation operation has been performed in order to avoid the generation of totally symmetric ramp edges which would be produced by direct linear smoothing. Then the blurry image has been contaminated by $10 \%$ impulsive noise (I) as shown Fig. 4 $\mathrm{d}$.

Figures 4b and 4 depict the output of the VMF when applied to the blurred and noisy test image. As can be noticed the VMF removes the impulse noise and preserves the blurred edges. This behavior is not present when inspecting the output of the GVMF filter, which is able to enhance image edges, (Fig. 45) while suppressing the impulsive noise, (Fig. 4f). It is worth noticing that the ramp edges tend to be converted into ideal step edges which separate piecewise constant image regions.

The ability of the GVMG to remove impulses while sharpening the color image edges by reducing their width is also depicted in Fig. 5 w which exhibits the row 60 of the blue channel of color test image shown in Fig. $4 \mathrm{~d}$. As seen in the magnified part of the main plot the spikes originating from the noise are removed and the ramp edges tend to be converted into step edges.

This filter behavior is also clearly seen in Fig. 6 which presents three dimensional representation of the blue channel of the artificial test images. The comparison of the GVMF technique with the standard VMF shows that the latter removes efficiently the impulse noise but preserves the blurred edges. The proposed filtering design replaces the impulses as efficiently as the VMF does but generates an 


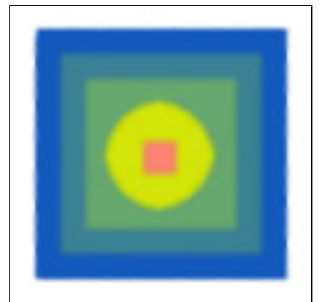

(a) test image

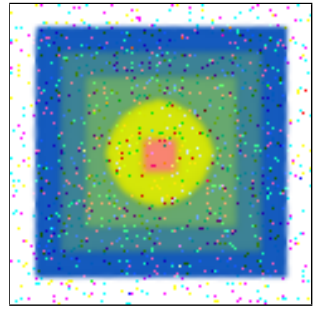

(d) noisy image

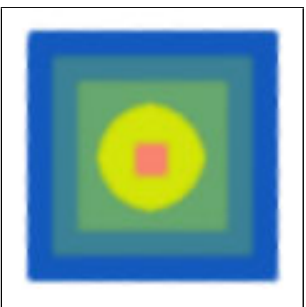

(b) $\mathrm{VMF}$

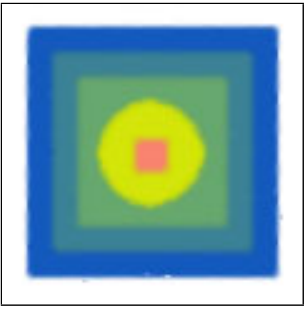

(e) VMF

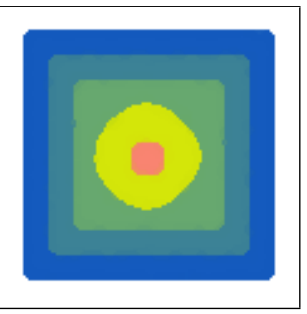

(c) GVMF, $\alpha=6$

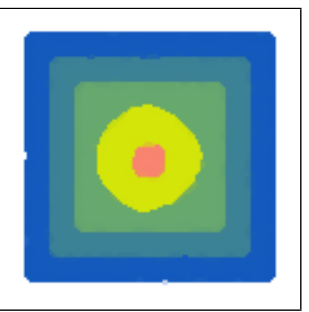

(f) GVMF, $\alpha=6$

Fig. 4. Edge enhancing and noise attenuating properties of the GVMF as compared with VMF: (a) blurry test image, (b) its VMF output, (c) GVMF with $\alpha=6$, (d) test image distorted by impulsive noise (noise model I, $p=0.1$ ), (d) VMF and (e) GVMF output with $\alpha=6$
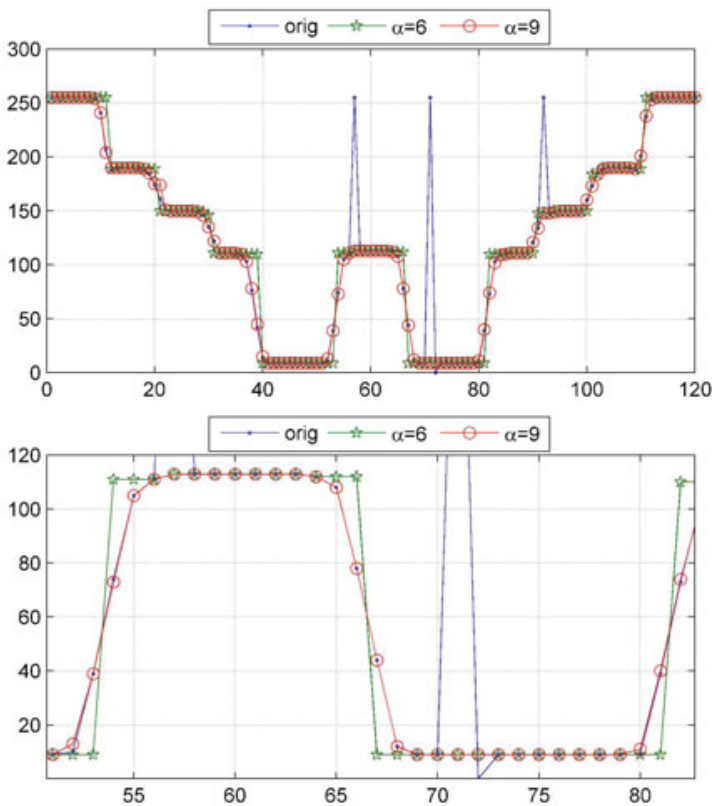

Fig. 5. Plot of the blue channel intensities (row 60) of the corrupted and enhanced synthetic test image depicted in Fig. $4 \mathrm{c}$ and below a zoomed part of the graph 


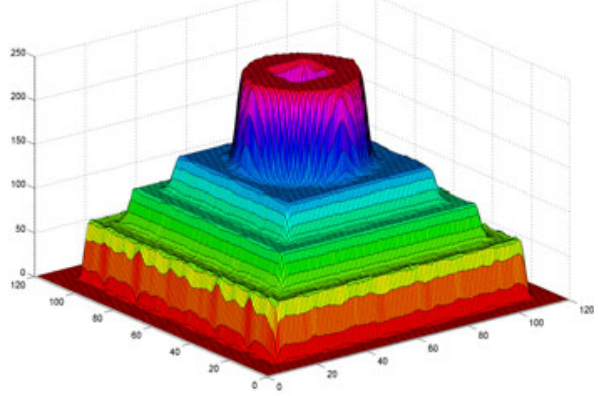

(a) test image

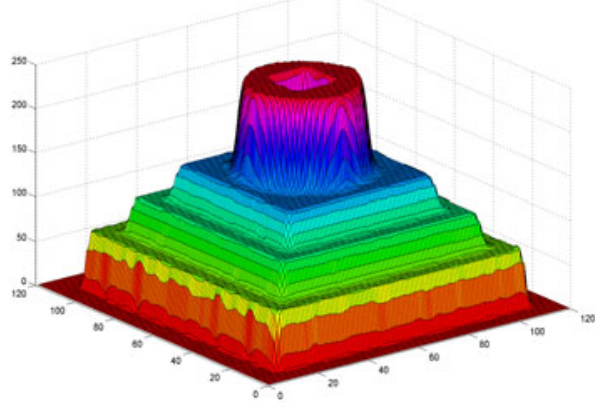

(c) $\mathrm{VMF}$

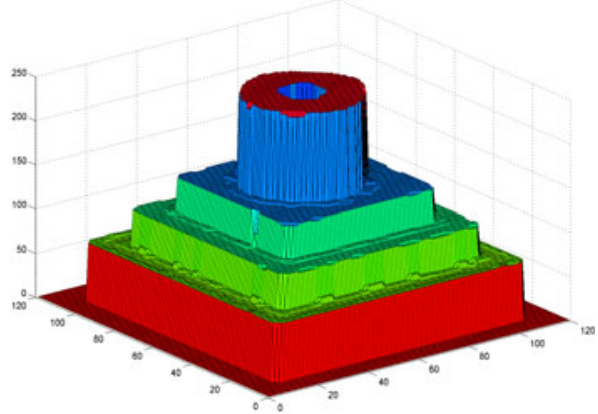

(e) GVMF, $\alpha=6$

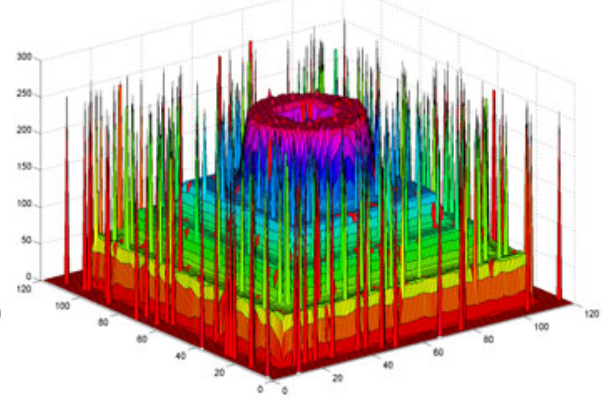

(b) noisy test image

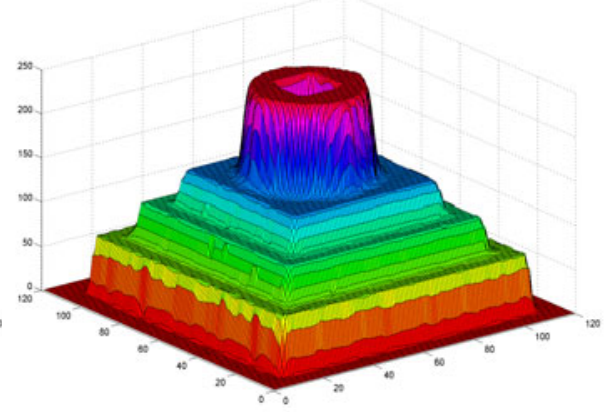

(d) VMF

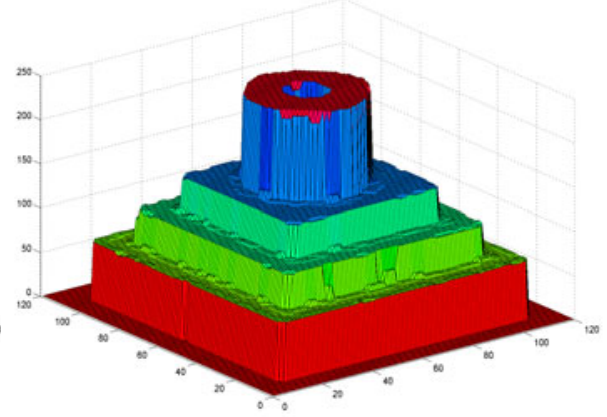

(f) GVMF, $\alpha=6$

Fig. 6. Visualization of the edge enhancing and noise reduction capabilities of the generalized VMF (GVMF) with fixed $\alpha=6$ : (a) 3D representation of the inverted blue channel of the test image depicted in Fig. 4a, (b) visualization of the inverted blue channel of the noisy test image shown in Fig. $4 \mathrm{~d},(\mathrm{c})$ and (d) the result of the VMF, (e) and (f) output of the GVMF 


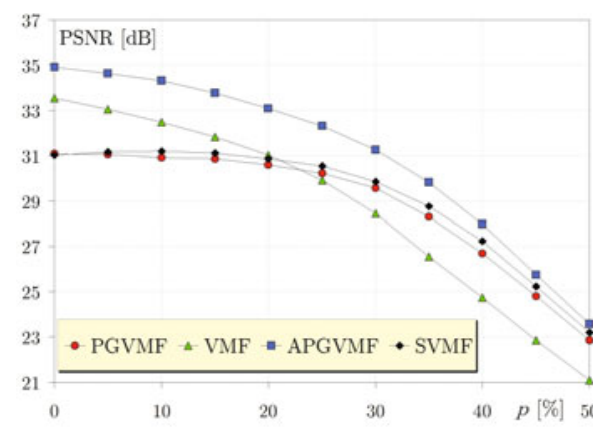

(a) noise model U

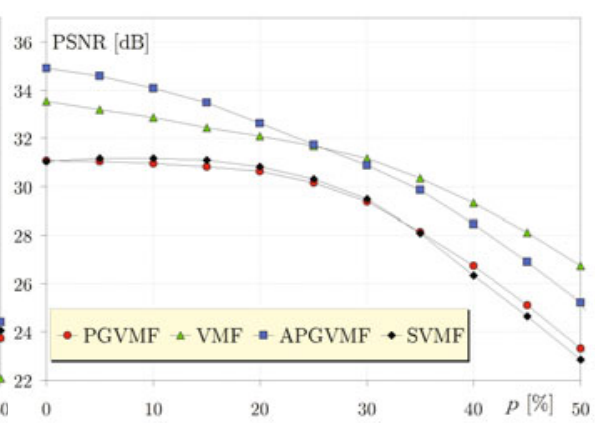

(b) noise model I

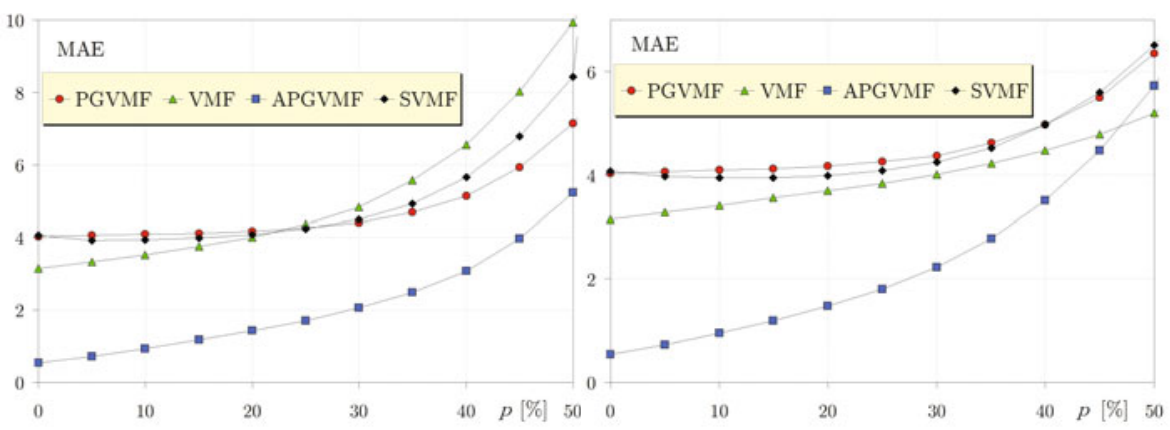

(c) noise model U

(d) noise model I

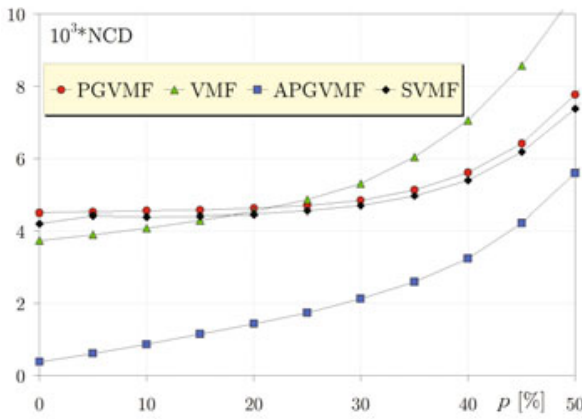

(e) noise model U

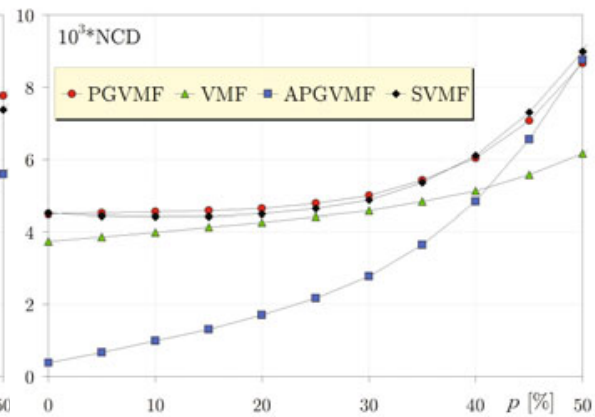

(f) noise model I

Fig. 7. Dependence of the PSNR (a, b), and MAE (c, d) and NCD (e, f) quality measures on the contamination intensities for the noise model $\mathrm{U}$ and I applied to the LENA test image filtered with AGVMF with $\delta=30$ as compared with other denoising techniques 


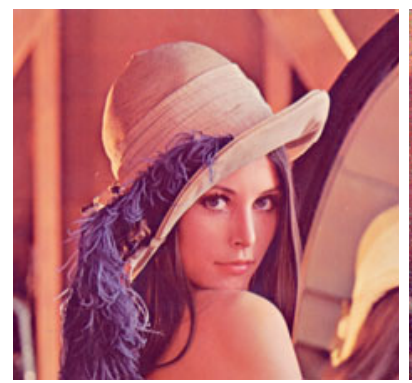

(a) test image

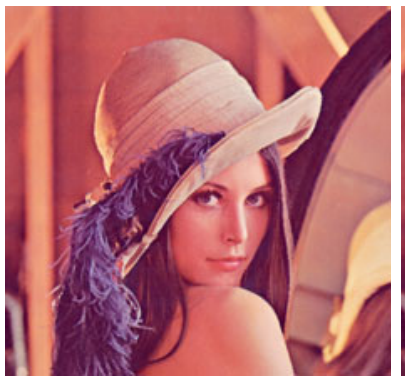

(d) AGVMF, $\delta=30$

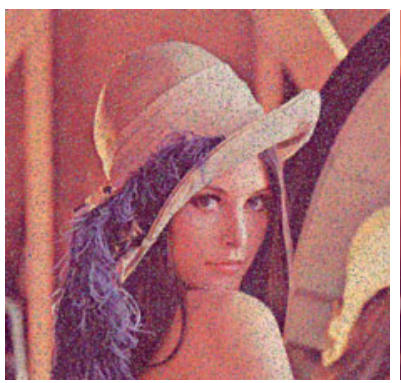

(b) noisy image

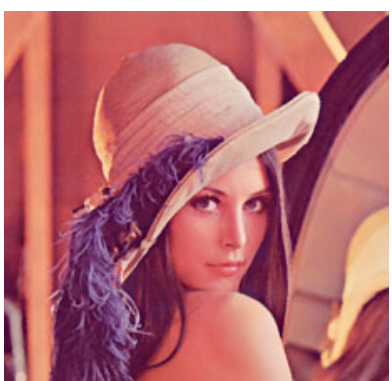

(e) GVMF $\alpha=6$

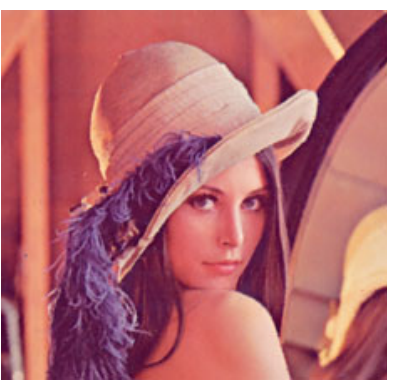

(c) $\mathrm{VMF}$

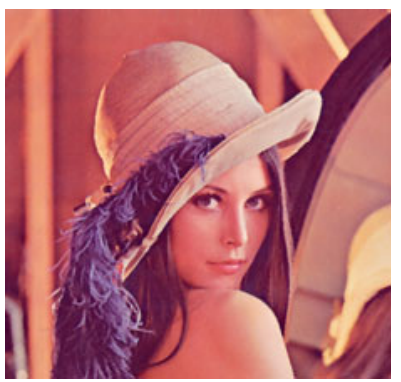

(f) SVMF

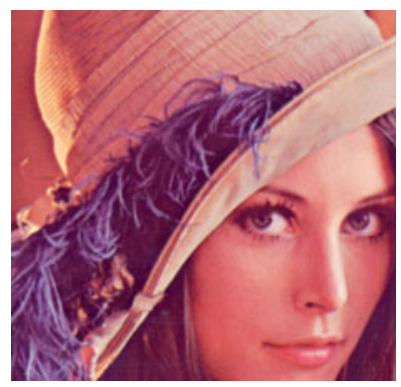

(g) test image

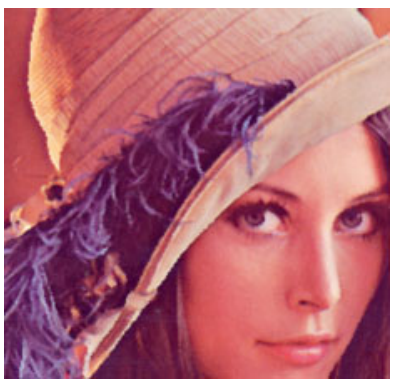

(j) AGVMF, $\delta=30$

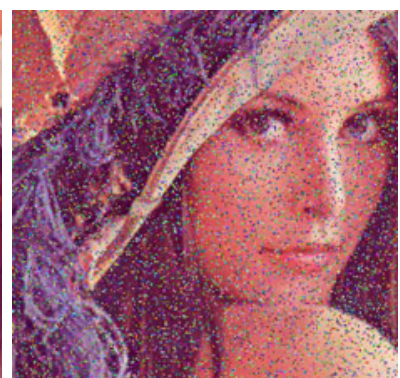

(h) noisy image

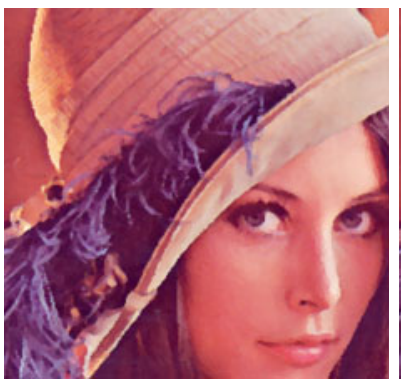

(k) GVMF $\alpha=6$

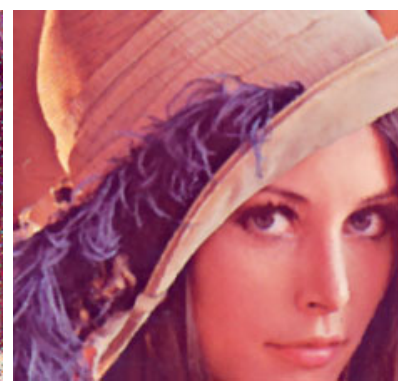

(i) $\mathrm{VMF}$

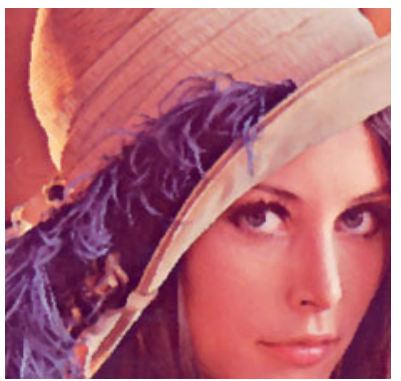

(l) SVMF

Fig. 8. Comparison of the proposed filtering technique with the standard VMF: (a) color test image, (b) test image distorted by $20 \%$ noise U, (c) VMF output, (d) AGVMF output with $\delta=30$, (e) GVMF with $\alpha=6$, (f) SVMF output 


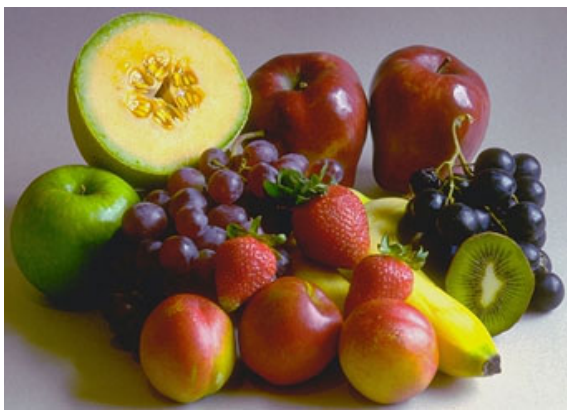

(a) test image FRUITS

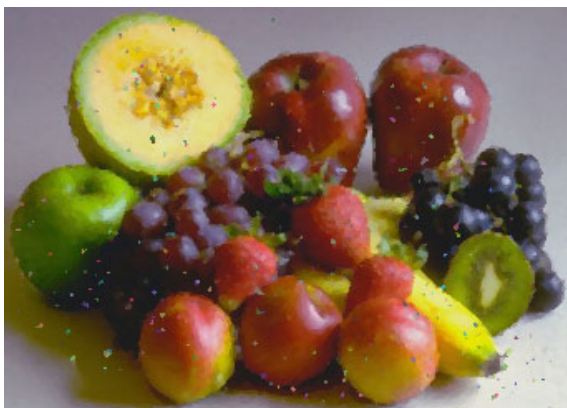

(c) VMF output, 2 iterations

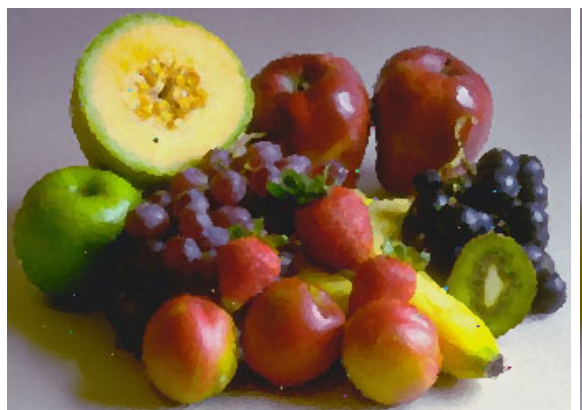

(e) GVMF output, 2 iterations, $\alpha=6$

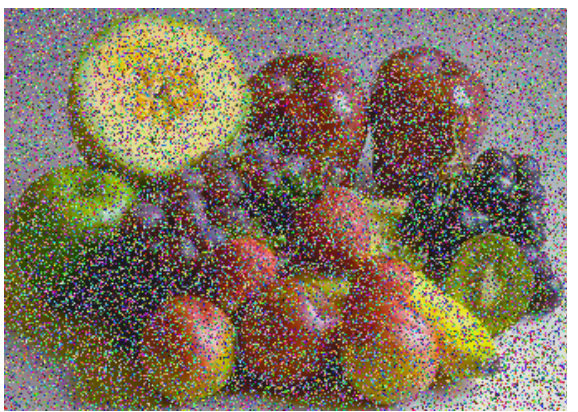

(b) noisy image, noise model $\mathrm{U}, p=0.4$

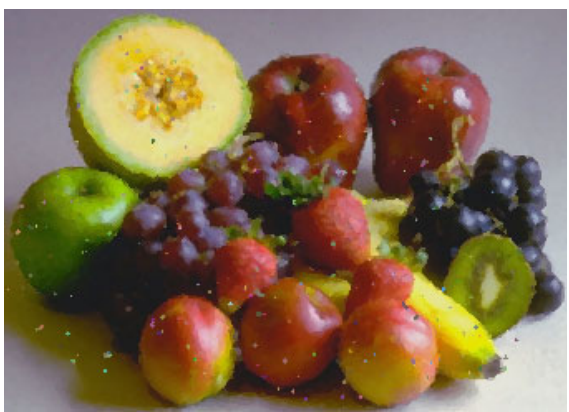

(d) DDF output, 2 iterations

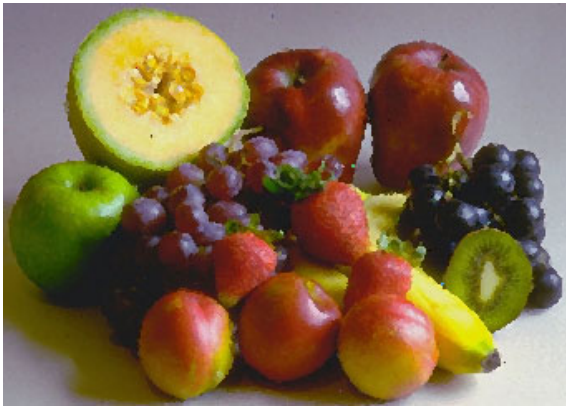

(f) AGVMF, 2 iterations, $\delta=30$

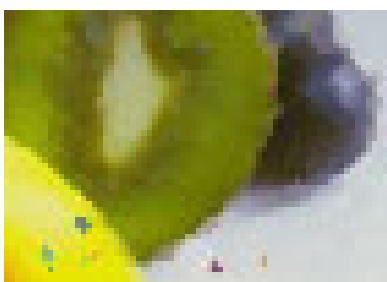

(g) VMF

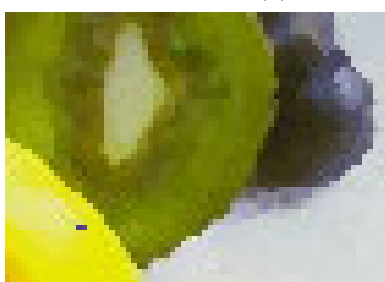

(h) GVMF

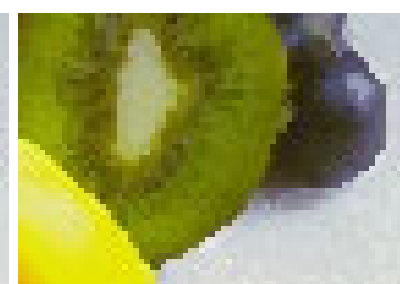

(i) AGVMF

Fig. 9. Efficiency of the proposed Adaptive Generalized VMF - AGVMF (f) as compared with the VMF (c), DDF [14, 15, (d) and GVMF (e) using a color test image (a) contaminated with $40 \%$ noise U (b). Below cropped and zoomed parts of the outputs of $\operatorname{VMF}(\mathrm{g}), \operatorname{GVMF}(\mathrm{h})$ and AGVMG with $\delta=30$ (i). 
image with sharp, almost ideal edges. Extensive experiments revealed that very similar results were obtained for images distorted by the uniform noise model.

The overall good noise reduction abilities of the proposed adaptive filtering design, are presented in Fig. 7 which show the dependance of the PSNR and MAE on the uniform noise (U) intensity $p$ when restoring the $L E N A$ noisy image. As can be observed the efficiency of the proposed adaptive GVMF (AGVMF) is superior to that of the Sharpening VMF (SVMF) proposed in [22] and the Generalized VMF (GVM F) with fixed parameter $\alpha=5$. As can be observed the efficiency of the proposed adaptive filter in terms of the used quality measures is lower for the impulsive noise I. This behavior is caused by the creation of clusters of similar pixels which are injected by the impulsive noise. For contamination intensity exceeding $30 \%$, the number of such clusters of noisy pixels is rapidly increasing and as a result the noisy pixels are preserved by the proposed filter.

Apart from the good denoising efficiency, the proposed adaptive filtering scheme has the unique ability to sharpen the edges present in the color images. This feature is visible in Fig. 8 which depicts the filtering results delivered by the new filter as compared with the VMF, GVMF and SVMF. As can be observed the new filtering design not only removes efficiently the impulses but also preserves image details due to the incorporated switching mechanism.

The detail preservation can be also observed when evaluating the output of the novel filter operating on a very noisy image depicted in Fig. 9. As can be seen the proposed filter removes the noise component while preserving fine image structures and significantly outperforms the basic filters like VMF, Directional Distance filter (DDF) [14, 15] and the GVMF with a fixed $\alpha$ parameter.

\section{Conclusions}

In the paper an adaptive filtering design for impulsive noise removal has been presented. The proposed adaptive scheme of choosing the optimal value of the peer group size used in the construction of the filter exhibits very good denoising properties outperforming the vector median based solutions. Extensive simulations revealed very good noise attenuation properties of the proposed filtering scheme combined with its unique ability to sharpen image edges while preserving image details. As a result, the novel class of filters exhibits very good noise reduction efficiency which combined with its edge enhancing properties makes the new filtering design an attractive tool for low level color image processing. The simplicity of the new algorithm and its computational speed, which is comparable to that of the VMF makes the new noise removal method very useful in the preprocessing of color images corrupted by impulse noise.

Acknowledgments. This work has been supported by the Polish Ministry of Science and Higher Education Development Grant OR 0000211. 


\section{References}

1. Plataniotis, K.N., Venetsanopoulos, A.N.: Color Image Processing and Applications. Springer, Heidelberg (2000)

2. Boncelet, C.G.: Image Noise Models. In: Bovik, A. (ed.) Handbook of Image and Video Processing, pp. 325-335. Academic Press, London (2000)

3. Zheng, J., Valavanis, K.P., Gauch, J.M.: Noise Removal from Color Images. Journal of Intelligent and Robotic Systems 7, 257-285 (1993)

4. Lukac, R., Smolka, B., Martin, K., Plataniotis, K.N., Venetsanopoulos, A.N.: Vector Filtering for Color Imaging. IEEE Signal Processing Magazine 22, 74-86 (2005)

5. Astola, J., Haavisto, P., Neuvo, Y.: Vector Median Filters. Proc. of the IEEE 78, 678-689 (1990)

6. Tang, K., Astola, J., Neuvo, Y.: Multichannel Edge Enhancement in Color Image Processing. IEEE Transactions on Circuits and Systems for Video Technology 4, 468-479 (1994)

7. Tang, K., Astola, J., Neuvo, Y.: Nonlinear Multivariate Image Filtering Techniques. IEEE Transactions on Image Processing 4, 788-798 (1995)

8. Leu, J.G.: Edge Sharpening Through Ramp Width Reduction. Image and Vision Computing 18, 501-514 (2000)

9. Schavemaker, J.G.M., Reinders, M.J.T., Gerbrands, J.J., Backer, E.: Image Sharpening by Morphological Filtering. Pattern Recognition 33, 997-1012 (2000)

10. Lin, W.S., Gai, Y.L., Kassim, A.A.: Perceptual Impact of Edge Sharpness in Images. IEE Proceedings - Vision, Image and Signal Processing 153, 215-223 (2006)

11. Kenney, C., Deng, Y., Manjunath, B.S., Hewer, G.: Peer Group Image Enhancement. IEEE Transactions on Image Processing 10, 326-334 (2001)

12. Deng, Y., Kenney, S., Moore, M.S., Manjunath, B.S.: Peer Group Filtering and Perceptual Color Image Quantization. Proc. of ISCAS 4, 21-24 (1999)

13. Pitas, I., Venetsanopoulos, A.N.: Nonlinear Digital Filters, Principles and Applications. Kluwer Academic Publishers, Boston (1990)

14. Karakos, D., Trahanias, P.E.: Generalized Multichannel Image Filtering Structures. IEEE Trans. on Image Processing 6, 1038-1045 (1997)

15. Trahanias, P.E., Karakos, D.G., Venetsanopoulos, A.N.: Directional Processing of Color Images: Theory and Experimental Results. IEEE Trans. on Image Processing 5, 868-881 (1996)

16. Astola, J., Kuosmanen, P.: Fundamentals of Nonlinear Digital Filtering. CRC Press, Boca Raton (1997)

17. Lukac, R.: Color Image Filtering by Vector Directional Order-Statistics. Pattern Recognition and Image Analysis 12, 279-285 (2002)

18. Lukac, R.: Adaptive Vector Median Filtering. Pattern Recognition Letters 24, 1889-1899 (2003)

19. Smolka, B., Plataniotis, K.N., Chydzinski, A., Szczepanski, M., Venetsanopulos, A.N., Wojciechowski, K.: Self-adaptive Algorithm of Impulsive Noise Reduction in Color Images. Pattern Recognition 35, 1771-1784 (2002)

20. Smolka, B., Lukac, R., Chydzinski, A., Plataniotis, K.N., Wojciechowski, K.: Fast Adaptive Similarity Based Impulsive Noise Reduction Filter. Real Time Imaging 9, 261-276 (2003)

21. Smolka, B., Chydzinski, A.: Fast Detection and Impulsive Noise Removal in Color Images. Real-Time Imaging 11, 389-402 (2005)

22. Lukac, R., Smolka, B., Plataniotis, K.N.: Sharpening Vector Median Filters. Signal Processing 87, 2085-2099 (2007) 